\title{
COMPACTAÇÃO DE UM LATOSSOLO VERMELHO CAUSADA PELO TRÁFEGO DO "FORWARDER"
}

\author{
Haroldo Carlos Fernandes² e Amaury Paulo de Souza ${ }^{3}$
}

\begin{abstract}
RESUMO - A mecanização tem sido, atualmente, um poderoso instrumento para suprimento das necessidades da indústria agroflorestal, estando presente nos processos de produção, colheita e transporte florestal. Esta utilização intensiva de máquinas pode, entretanto, acarretar danos ao solo, principalmente em termos de compactação, podendo refletir negativamente na produtividade. Tendo em vista a escassez de informações sobre a compactação do solo causada pelas máquinas florestais, o objetivo principal deste trabalho foi a avaliação dos níveis de compactação do solo causada pelo tráfego do trator florestal autocarregável (Forwarder). As variáveis físicas do solo analisadas foram a densidade e a resistência à penetração, tendo as amostras de solo sido retiradas em cinco pontos igualmente espaçados ao longo das trilhas, em três níveis de profundidades $(0-15 \mathrm{~cm}, 15-30 \mathrm{~cm}$ e $30-50 \mathrm{~cm})$. A análise estatística dos resultados de cada variável foi efetuada por meio da análise de variância para parcelas subdivididas, em que foram testados o efeito da máquina, o efeito da profundidade e o efeito da interação entre a máquina e a profundidade. A análise dos resultados obtidos permitiu constatar que o Forwarder provocou uma pequena compactação no solo, ou seja, incrementos de $0,06 \mathrm{~g} / \mathrm{cm}^{3}$ na densidade e $1,00 \mathrm{MPa}$ na resistência à penetração do solo. Os valores máximos da densidade do solo e resistência à penetração, depois da última passada da máquina, foram $1,12 \mathrm{~g} / \mathrm{cm}^{3}$ e 2,87 Mpa, respectivamente.
\end{abstract}

Palavras-chave: Compactação, solo florestal e máquinas.

\section{COMPACTION OF A DARK-RED LATOSOL CAUSED BY FORWARDER TRAFFIC}

\begin{abstract}
Mechanization is a powerful tool in the agro-forest industry, today, playing an important role in the processes of forest production, exploration and transportation. However, this intensive machinery utilization can cause severe damages to the soil, especially compaction, which can have a negative reflection on productivity. The main objective of this research was to evaluate the effects of soil compaction caused by forwarder traffic. The soil physical parameters analyzed were: soil density and soil penetration resistance, with the soil samples being taken at five sampling points, equally spaced along the exploration track, at three depth levels $(0-15 \mathrm{~cm}, 15-30 \mathrm{~cm}$ and 30-50 cm). Each variable was analyzed by variance analysis, applied in split plots with the following effects being evaluated: machine, depth, and machine-depth interaction. The result analysis showed that the forwarder caused a small soil compaction i.e., an average increase close to $0.06 \mathrm{~g} / \mathrm{cm}^{3}$ in soil density and $1.00 \mathrm{MPa}$ in soil penetration resistance. The maximum values of soil density and penetration resistance after machine traffic were, respectively, $1,12 \mathrm{~g} / \mathrm{cm}^{3}$ and 2,87 $\mathrm{MPa}$.
\end{abstract}

Key words: Compaction, forest soil and machinery.

1 Recebido para publicação em 30.1.2002.

Aceito para publicação em 12.5.2003.

Projeto desenvolvido com o auxílio da Fundação de Amparo à Pesquisa do Estado de Minas Gerais - FAPEMIG.

2 Prof. Adjunto - Dep. de Engenharia Agrícola da Universidade Federal de Viçosa - UFV, 36571-000 - Viçosa-MG. $<$ haroldo@ufv.br>; ${ }^{3}$ Prof. Titular, Dep. de Engenharia Florestal da UFV. 


\section{INTRODUÇÃOO}

Lira Filho (1992) mencionou que a compactação é um dos mais sérios danos causados ao solo, devido à exploração florestal.

O conhecimento da compactação do solo e de sua relação com o sistema de colheita de madeira são fontes importantes para o manejo adequado das condições físicas do solo, para melhorar a produção de madeira. $\mathrm{O}$ grau desejado de compactação depende do propósito a ser atingido. Por exemplo, os requisitos para tração e mobilidade são bem diferentes daqueles para infiltração e desenvolvimento das raízes (Cintra et al., 1983; Schafer et al., 1989).

De acordo com Scopel et al. (1992), os solos florestais podem ser compactados por animais em pastejo e também pelas raízes de árvores, porém mais significativos são os efeitos da mecanização. As máquinas empregadas na colheita, em geral, são muito pesadas e, combinadas com a movimentação e o levantamento de toras, exercem grandes pressões no solo.

As operações mecanizadas de colheita florestal intensificaram-se no início da década de 90. Desde então, o sistema de colheita florestal tem levado o solo a um processo acelerado de degradação, principalmente devido ao aumento do tráfego de máquinas, resultando em desequilíbrio de suas características físicas, químicas e biológicas e afetando progressivamente o seu potencial produtivo (Seixas, 2002). Segundo o autor, o tráfego de máquinas em uma floresta resulta em alguns impactos no ecossistema, ocasionando danos às árvores remanescentes, prejuízos à capacidade de rebrota das cepas e influência na qualidade dos recursos hídricos e na compactação do solo florestal.

Estudando a compactação em relação ao arraste de madeira com tratores agrícolas, MacDonagh et al. (1995) observaram incrementos de densidade do solo e de resistência à penetração em pontos de maior teor de água.

A compactação pode alterar o fluxo de água no solo, reduzir a produtividade do sítio e aumentar os níveis de erosão, uma vez que, geralmente, reduz a taxa de infiltração, aumentando o escorrimento superficial, pois a difusão da água depende do tamanho dos poros (Seixas, 2000).

Segundo Fenner (2002), o tráfego de máquinas prejudica o desenvolvimento e o crescimento de raízes $\mathrm{e}$, conseqüentemente, diminui o crescimento florestal, ou

R. Árvore, Viçosa-MG, v.27, n.3, p.279-284, 2003 seja, a produção de madeira. É necessário, então, que se desenvolvam estratégias com vistas a minimizar esse efeito, atuando sobre as suas causas.

Em relação à sensibilidade à compactação do solo em condições de casa de vegetação, árvores de Eucalyptus grandis tiveram o crescimento de raízes inibido quando a densidade do solo apresentou valores na faixa de 1,25 a $1,35 \mathrm{~g} / \mathrm{cm}^{3}$ (Borges et al., 1986).

Jakobsen e Greacem (1985), estudando a compactação causada por Forwarder em solos florestais com plantio de pinus, observou que os valores de resistência do solo à penetração eram acrescidos de $2 \mathrm{a} 3 \mathrm{kgf} / \mathrm{cm}^{2} \mathrm{a}$ cada passada da máquina e que a densidade do solo aumentava e a condutividade hidráulica diminuía com o número de passadas. Para trilhas de exploração mais antigas o mesmo não ocorreu.

O Forwarder é a máquina projetada para utilização na extração da madeira já cortada, de dentro da floresta para a periferia dos talhões, de modo a evitar o tráfego dos veículos de transporte dentro da floresta (Santos, 1991).

Sendo assim, o objetivo do presente trabalho foi avaliar os níveis de compactação do solo causados pelo tráfego do Forwarder, trabalhando em um Latossolo Vermelho.

\section{MATERIAL E MÉTODOS}

\subsection{Características Gerais das Áreas de Estudo}

O presente trabalho foi realizado no ano de 1996, em uma área de reflorestamento com Eucalyptus grandis de 7 anos, plantados no espaçamento de 3,0 x 1,5 m, situada na região de Guanhães-MG, com latitude de $18^{\circ} 46^{\prime} 48^{\prime \prime}$ (S), longitude Longitude (W Gr) e altitude de $750 \mathrm{~m}$.

\subsection{Seleção e Demarcação das Trilhas}

Para seleção das áreas utilizadas no experimento, demarcaram-se trilhas novas com $100 \mathrm{~m}$ de comprimento, $10 \mathrm{~m}$ de largura e declividade de $20 \%$, localizadas em uma área mais central do talhão.

\subsubsection{Máquina Utilizada}

A máquina utilizada na pesquisa foi um trator florestal autocarregável (Forwarder) marca ENGESA, 
modelo 510, trabalhando com toras de 0,124 m de diâmetro médio, 2,20 m de comprimento e número médio de 36 toras por pilha, cortadas pelo Harvester.

As principais especificações técnicas da máquina utilizada são: comprimento total $=9.410 \mathrm{~mm}$; largura $=$ $2.716 \mathrm{~mm}$; altura com carregador $=2.560 \mathrm{~mm}$; comprimento da plataforma de carga $=4.500 \mathrm{~mm}$; vão-livre do solo $=590 \mathrm{~mm}$; raio de giro $=10.000 \mathrm{~mm}$; peso em ordem de marcha $=100.000 \mathrm{~N}$; capacidade de subida em rampa $=60 \%$ e inclinação lateral máxima $=30 \%$.

O motor é de ciclo diesel, quatro-tempos, injeção direta, MWM D225, seis cilindros com potência nominal de $130 \mathrm{cv}(95,6 \mathrm{~kW})$ epneus 23,50 x 25 de 12 lonas.

A superfície de contato com o solo foi de $0,165 \mathrm{~m}^{2}$, o peso da máquina de $100.000 \mathrm{~N}$; carga média de $10.460 \mathrm{~N}$ e pressão de contato carregado por rodado de $0,152 \mathrm{MPa}$.

Na extração das toras a máquina subia vazia e retornava pela trilha central, demarcada no projeto, carregando as toras enleiradas nas linhas laterais pelo Harvester.

\subsection{Determinação dos Níveis de Compactação das Trilhas de Exploração}

\subsubsection{Amostragem do Solo}

Procedeu-se à coleta de solo em cinco pontos espaçados de, aproximadamente, $20 \mathrm{~m}$ ao longo do comprimento das trilhas demarcadas.

A coleta das amostras se deu antes e após a máquina ter retirado todo o volume de madeira existente na trilha, seguindo-se o ritmo normal de trabalho, sem nenhuma interferência externa.

As amostras foram retiradas em três faixas de profundidades do solo: $0-15 \mathrm{~cm} ; 15-30 \mathrm{~cm}$ e $30-50 \mathrm{~cm}$.

\subsection{Determinações Físicas das Amostras de Solo}

As determinações físicas das amostras de solo foram realizadas no laboratório de solos do Departamento de Solos da Universidade Federal de Viçosa.

O solo da região estudada foi classificado como um Latossolo Vermelho Eutrófico, textura argilosa.

\subsection{Variáveis Físicas Analisadas}

\subsubsection{Densidade do Solo (DS)}

As amostras de solo para determinação da densidade do solo (DS) foram retiradas em cinco pontos, espaçados aproximadamente de $20 \mathrm{~m}$ ao longo das trilhas, em três profundidades.

O método utilizado foi o de impermeabilização dos torrões com parafina, segundo Mello \& Teixeira (1973).

\subsubsection{Resistência à Penetração do Solo}

Para determinação da resistência do solo à penetração, utilizou-se um penetrógrafo da marca SOIL CONTROL, modelo SC-60, com uma haste de $600 \mathrm{~mm}$ de comprimento, 9,53 $\mathrm{mm}$ de diâmetro, equipada com um cone de $129,3 \mathrm{~mm}^{2}$ de área de base, $12,83 \mathrm{~mm}$ de diâmetro e $30^{\circ}$ de ângulo de vértice. As determinações foram feitas conforme a recomendação da ASAE S 313, mencionada por Balastreire (1987). Nos testes procurouse utilizar uma velocidade de penetração constante.

Foram determinados valores em cinco pontos, espaçados aproximadamente de $20 \mathrm{~m}$ ao longo da trilha e analisados para as três profundidades preestabelecidas, com os valores transformados em MPa.

\subsection{Delineamento Estatístico}

A análise estatística dos resultados de cada variável foi efetuada por meio da análise de variância para parcelas subdivididas, conforme Snedecor \& Cochran (1980), considerando a diferença $|\mathrm{Y}|=$ antes-depois (para algumas variáveis) e $|\mathrm{Y}|=$ depois-antes, com o objetivo de verificar diferenças provocadas pela passada da máquina em três profundidades (0-15, 15-30 e 30-50).

Foram testados:

1. efeito de máquina;

2. efeito de interação entre a máquina e a profundidade; e

3. efeito de profundidade.

No caso de efeitos significativos $(\mathrm{p}<0,05)$ foram efetuados contrastes entre pares de médias, com o cálculo da diferença mínima significativa (dms) pelo método de Tukey, para $\alpha=0,05$.

Nos casos em que $0,05<\mathrm{p}<0,10$, foram referidas tendências à significância.

R. Árvore, Viçosa-MG, v.27, n. 3, p.279-284, 2003 


\section{RESULTADOS E DISCUSSÃO}

\subsection{Densidade do Solo}

No Quadro 1 estão os valores da densidade do solo antes e depois das passadas da máquina.

Quadro 1 - Resultados da densidade do solo $\left(\mathrm{g} / \mathrm{cm}^{3}\right)$, considerando a profundidade da amostra $(\mathrm{cm})$

Table 1 - Soil density $\left(\mathrm{g} / \mathrm{cm}^{3}\right)$ results, considering sample (cm) depth

\begin{tabular}{|c|c|c|c|c|c|}
\hline \multicolumn{6}{|c|}{ Profundidade da Amostra } \\
\hline \multicolumn{2}{|c|}{$0-15$} & \multicolumn{2}{|c|}{$15-30$} & \multicolumn{2}{c|}{$30-50$} \\
\hline $\mathrm{A}$ & $\mathrm{D}$ & $\mathrm{A}$ & $\mathrm{D}$ & $\mathrm{A}$ & $\mathrm{D}$ \\
\hline 1,05 & 1,12 & 1,07 & 1,04 & 1,05 & 1,12 \\
1,05 & 1,07 & 1,06 & 1,07 & 1,06 & 1,12 \\
1,01 & 1,09 & 1,04 & 1,07 & 1,07 & 1,07 \\
1,03 & 1,07 & 1,04 & 1,05 & 1,07 & 1,08 \\
1,01 & 1,10 & 1,04 & 1,07 & 1,10 & 1,11 \\
\hline
\end{tabular}

$\mathrm{A}=$ amostra retirada antes da passada da máquina.

$\mathrm{D}=$ amostra retirada depois da última passada da máquina.

Com os dados obtidos foi efetuada a análise de variância, em que se avaliou o efeito da máquina, da profundidade e da interação máquina $x$ profundidade, chegando aos resultados:

- efeito de máquina:

$$
\mathrm{F}=307,69, \mathrm{p}<0,001
$$

Ocorreu um incremento na densidade do solo. - efeito de interação:

$$
\mathrm{F}=2,68 ; \mathrm{p}<0,05
$$

Ocorreu efeito significativo de interação entre máquina e profundidade.

- efeito de profundidade:

$$
\mathrm{F}=0,81, \mathrm{p}>0,10
$$

Não foi constatado o efeito da profundidade.

De modo geral, constatou-se que a densidade do solo aumentou com a profundidade. Estes resultados estão de acordo com a afirmação de Brandy, citado por Rando (1981), que relatou que muitas vezes, com o aumento da profundidade, ocorre uma tendência natural de elevação da densidade do solo, devido à menor quantidade de matéria orgânica, menor agregação, bem como maior compactação provocada pelo peso das camadas sobrejacentes.

\subsection{Resistência à Penetração}

No Quadro 2 estão os valores da resistência à penetração antes e depois das passadas da máquina. Entre parênteses estão os valores dos teores de água.

Com os dados obtidos foi efetuada a análise de variância, em que se avaliou o efeito da máquina, da profundidade $\mathrm{e}$ da interação máquina $\mathrm{x}$ profundidade, chegando aos resultados:

- efeito de máquina:

$$
\mathrm{F}=10,633 ; \mathrm{p}<0,001
$$

Ocorreu um acréscimo nos valores.

- efeito de interação:

$$
\mathrm{F}=0,924 ; \mathrm{p}<0,01
$$

\begin{tabular}{|c|c|c|c|c|c|}
\hline \multicolumn{6}{|c|}{ Profundidade da Amostra $(\mathrm{cm})$} \\
\hline \multicolumn{2}{|c|}{$0-15$} & \multicolumn{2}{|c|}{$15-30$} & \multicolumn{2}{|c|}{$30-50$} \\
\hline A & $\mathrm{D}$ & A & $\mathrm{D}$ & A & D \\
\hline $1,88(12,7)$ & $2,79(12,1)$ & $1,97(12,9)$ & $2,48(13,1)$ & $1,93(14,5)$ & $1,62(14,1)$ \\
\hline $1,87(13,8)$ & $2,81(12,9)$ & $1,92(13,7)$ & $2,37(13,9)$ & $1,98(14,1)$ & $1,69(14,3)$ \\
\hline $1,79(13,1)$ & $2,87(13,6)$ & $1,83(14,3)$ & $2,39(14,1)$ & $1,89(15,2)$ & $1,59(15,0)$ \\
\hline $1,80(12,8)$ & $2,85(13,1)$ & $1,86(13,1)$ & $2,49(12,9)$ & $1,98(14,3)$ & $1,72(14,2)$ \\
\hline $1,81(11,7)$ & $2,83(11,5)$ & $1,92(13,0)$ & $2,27(13,8)$ & $2,07(14,4)$ & $1,73(14,1)$ \\
\hline
\end{tabular}

Quadro 2 - Resultados de resistência à penetração $(\mathrm{MPa})$, considerando a profundidade da amostra $(\mathrm{cm})$ e o teor de água $(\%)$

Table 2 - Penetration resistence (MPa) results, considering sample depth (cm) and water content (\%)

$\mathrm{A}=$ amostra retirada antes da passada da máquina e $\mathrm{D}=$ amostra retirada depois da última passada da máquina 
Ocorreu efeito significativo de interação entre máquina e profundidade.

- efeito de profundidade:

A diferença mínima significativa foi igual a 0,199.

Houve a seguinte relação entre as faixas de profundidades: 0-15 > 15-30 >30-50.

As diferenças entre os valores de resistência à penetração antes e depois das passadas foram altas pelo fato de essa máquina, para retirar todo o volume de madeira preestabelecido ao longo da trilha, precisar efetuar um grande número de passadas. Mantovani (1987) relatou que a energia de compactação é determinada pelo número de passadas da máquina sobre o solo e que as primeiras passadas são as que causam maiores danos ao solo.

De acordo com Keilen (1992), uma vez que se trabalhou sem a camada de galhos sobre o solo, a compactação é maior na primeira passada, aumentando gradativamente após cada nova passada.

\section{CONCLUSÕES}

Nas condições em que o experimento foi conduzido, chegou-se às seguintes conclusões:

- A densidade do solo e a resistência à penetração foram eficientes para determinação dos níveis de compactação dos trilhos.

- A utilização do rodado duplo contribuiu para diminuir a diferença nos valores antes e depois das passadas.

- O maior incremento nos valores da resistência e penetração foi observado na profundidade de $0-15 \mathrm{~cm}$.

- Os valores máximos de densidade do solo e resistência à penetração, depois da última passada da máquina, foram $1,12 \mathrm{~g} / \mathrm{cm}^{3}$ e 2,87 Mpa, respectivamente.

- Para atenuar a compactação no solo, as máquinas usadas na colheita florestal devem utilizar os pneus BPAF, com baixa pressão e alta flutuação.

\section{REFERÊNCIAS BIBLIOGRÁFICAS}

BALASTREIRE, L. A. Máquinas agrícolas. São Paulo: Manole, 1987. 307 p.
BORGES, E. N. Resposta da soja e do eucalipto à camadas compactadas de solo. 1986. 54 f. Dissertação (Mestrado em Solos e Nutrição de Plantas) - Universidade Federal de Viçosa, Viçosa, 1986.

CINTRA, F. L. D.; MIELNICZUK, J.; SCOPEL,

I. Caracterização do impedimento mecânico em um Latossolo Roxo do Rio Grande do Sul. Revista Brasileira da Ciência do Solo, v. 7, p. 323-327, 1983.

FENNER, P. T. Compactação do solo. In: FENNER, P. T. Colheita Florestal. Viçosa: Universidade Federal de Viçosa, 2002. p. 375-396.

JAKOBSEN, B. F; GREACEN, E. L. Compaction of sandy forest soils by Forwarder operations. Soil \& Tillage Research, v. 5., n. 1., p. 55-70, 1985.

KEILEN, K. Estudo da alteração do solo pelo trânsito intensivo. In: SEMINÁRIO DE ATUALIZAÇÃO SOBRE SISTEMAS DE EXPLORAÇÃO E TRANSPORTE FLORESTAL, 7. 1992, Curitiba. Anais... Curitiba: Fundação de Pesquisas Florestais do Paraná, Universidade Federal de Viçosa, 1992. p. 217-220.

LIRA FILHO, J. A. Interferências ambientais na exploração de florestas plantadas em regiões acidentadas do Vale do Rio Doce-MG. 1992. 97 f. Dissertação (Mestrado em Ciências Florestais) - Universidade Federal de Viçosa, Viçosa, 1992.

MAcDONAGH, P.M. et al. A compactação do solo em relação a oportunidade de arraste de madeira com tratores agrícolas. In: CONGRESSO BRASILEIRO DE ENGENHARIA AGRÍCOLA, 24, 1995, Viçosa. Anais... Viçosa: Sociedade Brasileira de Engenharia Agrícola, 1995. p. $1-20$.

MANTOVANI, E. C. Compactação do solo. Informe Agropecuário, v. 13, n. 17, p. 52-55, 1987.

MELLO, V. S. B.; TEIXEIRA, A. H. Mecânica dos solos: fundações e obras de terra. São Carlos: Engenharia de São Carlos, Universidade de São Paulo, 1973. 205 p.

SANTOS, G. S. A evolução de forwarders e harvesters na mecanização florestal. In: SIMPÓSIO BRASILEIRO SOBRE EXPLORAÇÃO E TRANSPORTE FLORESTAL, 1., 1991, Belo Horizonte. Anais... Belo Horizonte: Sociedade de Investigações Florestais, Universidade Federal de Viçosa, 1991. p. 127-142.

R. Árvore, Viçosa-MG, v.27, n.3, p.279-284, 2003 
SCHAFER, R. L. et al. A rationale for modeling soil compaction behavior: a mechanic engineering approach. American Society of Agricultural Engineering, n. 89, p. $1097,1989$.

SCOPEL, I. et al. Riscos de compactação do solo na produção florestal. In: SEMINÁRIO DE ATUALIZAÇÃO SOBRE SISTEMAS DE EXPLORAÇÃO E TRANSPORTE FLORESTAL, 7., 1992, Curitiba. Anais... Curitiba: Fundação de Pesquisas Florestais do Paraná, Universidade Federal de Viçosa, 1992. p. 172-193.
SNEDECOR, G. W.; COCHRAN, W. S. Statistical methods. 7.ed. Ames: Iowa State University Press, 1980. 550p.

SEIXAS, F. Olho na Terra. Revista Cultivar Máquinas, v. 2, n. 7, p. 15-17, 2002.

SEIXAS, F. Compactação do solo devido à colheita de madeira. 2000. $75 \mathrm{f}$. Tese (Livre - docência) - Escola Superior de Agricultura Luiz de Queiroz, Piracicaba, 2000. 